Original Paper http://ajol.info/index.php/ijbcs http://indexmedicus.afro.who.int

\title{
Insectes ravageurs et champignons parasites associés au dépérissement des peuplements de Tectona grandis (teck) régénérés à Téné, zone semi-décidue de Côte d'Ivoire
}

\author{
Bianuvrin Noël Boué VOUI BI ${ }^{1 *}$, Kanga Anatole N'GUESSAN ${ }^{2}$, \\ Koffi Fernand Jean Martial KASSI ${ }^{3}$, Foua Alponse TAPE BI ${ }^{4}$ et Kagoyire KAMANZI ${ }^{1}$ \\ ${ }^{1}$ Université Félix Houphouët-Boigny, UFR Biosciences, Laboratoire de Botanique, \\ 22 BP 582 Abidjan 22, Côte d'Ivoire. \\ ${ }^{2}$ Programme Forêt et Environnement, Centre National de Recherche Agronomique (CNRA), \\ 08 BP 33 Abidjan 08, Côte d'Ivoire. \\ ${ }^{3}$ Université Félix Houphouët-Boigny, UFR Biosciences, Laboratoire de Physiologie Végétale, \\ 22 BP 582 Abidjan 22, Côte d'Ivoire. \\ ${ }^{4}$ Centre de Gestion d'Anguédédou, Société de Développement des Forêts (SODEFOR), \\ 01 BP 3770 Abidjan 01, Côte d'Ivoire. \\ *Auteur correspondant, E-mail : noelboue@ gmail.com ; \\ Tél. : (225) 07506549 ; Adresse : 09 BP 433 Abidjan 09 (République de Côte d'Ivoire).
}

\section{RESUME}

Les rejets de souche de teck sont l'objet d'attaque par des ravageurs et champignons capables de freiner leur croissance et développement. L'objectif est d'identifier les insectes ravageurs et les champignons parasites associés au dépérissement des rejets de teck régénérés à partir des souches. Au plan méthodologique, les ravageurs ont été caractérisés par repérage et exploration des dégâts corticaux et sous-corticaux des rejets de teck. Les champignons ont été isolés à partir des racines de rejets présentant des symptômes d'infection fongique. Les résultats ont mis en évidence la présence des termites ( $g$. Ancistrotermes), des criquets puants (Zonocerus variegatus) et des coléoptères (Xyleborus aquilus). L'isolement et l'identification fongiques ont montré les espèces, Phellinus noxius, Fusarium verticilioides et les genres, Fusarium, Trichoderma, Penicillium et Aspergillus. Phellinus noxius, reconnu comme agent de pourriture brune racinaire provoque le dépérissement puis la mort des rejets de teck.

(C) 2015 International Formulae Group. All rights reserved.

Mots clés : Rejet de souche de teck, termites, criquets, coléoptères, champignons, dégâts.

\section{Ravager insects and fungi associate to dieback of stand of Tectona grandis (teak) regenerate at Téné, semi deciduous zone of Côte d'Ivoire}

\begin{abstract}
Sprouts of tools were attacked by ravagers and fungi capable to stop their growth and development. The objective of this work is to identify ravager insects and parasitizes fungi associate to dieback of sprouted from
\end{abstract}


tools of Teak. Methodologically, ravagers were characterized by search and exploration of cortical and subcortical damages of teak. Fungi were isolated from roots of sprout of stool presenting symptom of fungical infection. Results showed the presence of termites ( $g$. Ancistrotermes), stinking crickets (Zonocerus variegatus) and beetles Xyleborus aquilus). Isolation and identification revealed the presence of fungal species Phellinus noxius, Fusarium verticilioides and genius, Fusarium, Trichoderma, Penicillium and Aspergillus. Phellinus noxius, known like agent of brown root rot incite dieback and sprouts death.

(C) 2015 International Formulae Group. All rights reserved.

Keywords: Sprouted from tools of teak, termites, crickets, beetles, fungi, damage.

\section{INTRODUCTION}

Avec près de $33 \%$, la Côte d'Ivoire a l'un des taux de déforestation le plus élevé au monde (FAO, 2007). Afin de freiner cette exploitation abusive et sauvage, des programmes de gestion raisonnée et d'aménagement durable ont été initiés. Ces programmes, autrefois basés sur l'enrichissement en essences de valeur des forêts naturelles, n'ont pas endigué le phénomène de déforestation. Aussi, vu les résultats mitigés, des campagnes de reboisement industriel intensif basées sur l'utilisation d'espèces à vocation bois d'œuvre, feu et service sont initiées aujourd'hui (Yedmel, 2004). Ainsi, environ 150000 hectares de forêts artificielles constituées principalement de Tectona grandis, Gmelina arborea, Terminalia superba et de Cedrela odorata ont été reboisés (Koné, 2012 ; Voui Bi, 2015). Les reboisements en teck consistaient à planter après le défrichement total des parcelles. Mais la disponibilité de terres et les coûts de production, très onéreux, ne permettent pas d'atteindre les résultats escomptés. Parmi les alternatives, on note la création des peuplements par le traitement en taillis (Voui Bi et al., 2012).

Le taillis est une méthode de régénération rapide qui permet de réduire la pénibilité du travail ainsi que les surfaces cultivables. A cet égard, la régénération à partir des sujets sains, en quantité suffisante est un préalable indispensable à la réussite de toute action de reboisement. A Téné, zone de reboisement semi-décidue de Côte d'Ivoire, les peuplements de teck régénérés à partir des souches font l'objet d'attaque des ravageurs et parasites qui provoquent leur dépérissement. Chez le teck, le dépérissement touche en moyenne dans les plantations jeunes et adultes 1 à $25 \%$ des arbres et 50 à $80 \%$ dans les plantations de plus de 30 ans (CNRA, 2013). De ce fait, il s'avère opportun, d'identifier les contraintes liées à la régénération du teck. L'objectif de ce travail est de rechercher les pathogènes susceptibles de gêner la croissance et le développement des rejets de teck afin de mettre en place des stratégies de protection des peuplements.

\section{MATERIEL ET METHODES}

\section{Site d'étude}

L'étude a été réalisée sur 2 sites dans la forêt de Téné (Oumé). Le premier est situé entre $5^{\circ} 24^{\prime}$ de longitude Ouest et $6^{\circ} 29^{\prime}$ de latitude Nord. Le deuxième est localisé entre $5^{\circ} 24^{\prime}$ de longitude Ouest et $6^{\circ} 27^{\prime}$ de latitude Nord (Figure 1). Le climat, de type subéquatorial, comprend quatre saisons: deux saisons pluvieuses (mars-juin et septembreoctobre) et deux saisons sèches (décembrefévrier et juillet-août). Les précipitations enregistrées de 2001 à 2010 présentent une moyenne annuelle de $1176,31 \mathrm{~mm}$. Les températures moyennes annuelles varient de $24,59{ }^{\circ} \mathrm{C}$ à $25,80{ }^{\circ} \mathrm{C}$. L'humidité relative moyenne atteint $78,97 \%$. Le sol est de type ferralitique faiblement ou moyennement désaturé. Le relief est formé d'un plateau faiblement ondulé incliné vers l'Est, en 
direction du fleuve Bandama. La végétation est du domaine guinéen et principalement au secteur mésophile (Yedmel, 2004).

\section{Matériel d'étude}

Le matériel végétal était constitué des racines et écorces de Tectona grandis (teck). Les peuplements initiaux sont des futaies pures et équiennes âgées de 20 ans (site 1) et 25 ans (site 2). Les prélèvements ont été effectués sur des rejets de souches de teck régénérés après une coupe d'exploitation. Les écartements à la plantation étaient de 2,5 x 2,5 m (1600 pieds/ha). Le matériel animal comprenait des insectes ravageurs associés aux rejets de souche de teck.

\section{Prélèvement des échantillons}

Le prélèvement des échantillons a été effectué en parcourant les lignes de plantation des rejets de teck. Les rejets de souche présentant des signes de dépérissement ont été inventoriés et répertoriés. Des écorçages de tronc en vue d'une description macroscopique ont été réalisés sur les sujets symptomatiques. Des prélèvements racinaires en vue d'une analyse microscopique au niveau du pivot ont été menés. Les prélèvements ont été effectués en dégageant une partie de la masse de terre entourant les racines. A l'aide d'une machette et d'une pioche, les pivots des rejets dépéris ont été coupés à la base et mis dans des sachets stériles puis envoyés au laboratoire de Physiologie Végétale de l'Université Félix Houphouët-Boigny. Les racines ont été lavées pendant 10 minutes à l'eau courante afin de les débarrasser de leur gangue de terre puis 3 fois à l'eau distillée stérile. Les organes ont été découpés en morceaux cylindriques à l'aide d'une machette dont la lame a été préalablement stérilisée à l'aide d'alcool $90^{\circ}$. A l'aide d'un scalpel stérilisé plongé dans l'alcool puis flambé, les organes ont été découpés en des fragments de 3 à $5 \mathrm{~cm}$ environ et regroupées en deux lots. Les organes issus du premier lot sont prélevés au niveau de l'écorce et ceux du deuxième au niveau du cylindre central. Ces organes ainsi préparés ont servi à l'isolement des champignons pathogènes.

\section{Isolement des champignons}

L'isolement des champignons a été réalisé selon la méthode de Rapilly (1968). Les racines de teck ont été d'abord lavées à l'eau de robinet. Les organes ainsi obtenus ont été désinfectés à l'hypochlorite de sodium à $5 \%$ pendant $3 \mathrm{~min}$, puis rincées 3 fois pendant 3 minutes à l'eau distillée stérile afin d'éliminer les traces de désinfectant. Les organes ont été ensuite essorés dans du papier buvard stérile. Les étapes ci-après ont servi à l'isolement définitif.

\section{Préparation du milieu de culture}

Le milieu Potato Dextrose Agar (PDA) a été préparé en additionnant $20 \mathrm{~g}$ de purée de pomme de terre, $20 \mathrm{~g}$ d'Agar et $20 \mathrm{~g}$ de saccharose. L'ensemble a été transvasé dans un erlenmeyer de $1 \mathrm{~L}$ et le volume ajusté à $1 \mathrm{~L}$ avec de l'eau distillée puis autoclavé à $121^{\circ} \mathrm{C}$ sous une pression d'un bar pendant $20 \mathrm{~min}$. Le milieu a été ensuite refroidi à température ambiante du laboratoire pour atteindre $40{ }^{\circ} \mathrm{C}$. Il a été ensuite ajouté $350 \mu \mathrm{l}$ d'acide citrique à $0,3 \mathrm{mg} / \mathrm{l}$; le tout a été mélangé sous agitation magnétique et coulé dans des boîtes de Pétri à raison de $17 \mathrm{ml}$ par boîte. Ce milieu était destiné à l'isolement de la mycoflore totale.

\section{Incubation}

Les fragments d'organes de teck ont été ensemencés sur le milieu de culture PDA préalablement coulée dans des boîtes de Pétri stériles. Les explants ont été ensuite déposés selon le type sur les différents milieux de culture déjà solidifiés à raison de 5 explants par boîte de Pétri. Les boîtes de pétri ont été scellées avec un film étirable et étiquetées. Ces boîtes ont été ensuite mises dans une chambre d'incubation à l'obscurité pendant 3 jours. Les colonies mycéliennes obtenues ont été repiquées sur de nouveaux milieux PDA pour être purifiées. 


\section{Purification}

Les purifications ont été faites au fur et à mesure que les champignons apparaissaient en les repiquant sur un nouveau milieu PDA. Des repiquages successifs accompagnés d'observations microscopiques ont permis d'obtenir des cultures pures de champignons. La conservation de ces micro-organismes a été faite au congélateur à $-10^{\circ} \mathrm{C}$.

\section{Observations macroscopique et microscopique}

Les observations macroscopiques ont été faites sur les champignons purifiés. Elles ont permis de relever les aspects culturaux : la forme et la couleur des différents champignons. L'examen microscopique des fragments et de diverses cultures développées ont permis l'identification de la mycoflore. Ainsi, le montage d'un fragment de chaque champignon obtenu a été effectué entre lame et lamelle dans une goutte de bleu de coton lactique. Ces observations microscopiques ont permis de déterminer la forme des spores, la coloration et le cloisonnement ou non du mycélium. Les espèces fongiques ont été ensuite identifiées à l'aide de la clé d'identification de Barnett et Hunter (1960).

\section{Autres pathogènes}

En plus des pathologies d'ordre fongique, les études se sont également orientées vers la recherche d'agents de l'entomofaune. De ce fait, des signes d'attaque d'insectes, notamment des piqures, des sciures, des sécrétions ou exsudats ont été recherchés. La méthode a consisté d'une part, à repérer et à explorer les dégâts corticaux et sous-corticaux pour les caractériser. Les ravageurs (termites, criquets, charançons) prélevés ont été conservés dans des Piluliers étiquetés, contenant de l'alcool 70 degrés. Au laboratoire de Zoologie (Biologie Animale), les échantillons ont été triés puis identifiés.

\section{RESULTATS}

Aspect général des rejets de teck en dépérissement

\section{Symptômes d'attaque aérienne}

Les feuilles des rejets symptomatiques sont petites, épaisses, décolorées puis desséchées. Le dessèchement atteint les branches latérales, puis la tige principale (Figures $2 \mathrm{~A}$ et $2 \mathrm{~B}$ ). Les tiges complètement dépéries pourrissent et avec le temps, des champignons saprophytes y poussent (Figure 2C). Dans certains cas, les feuilles médianes et basales des rejets en début de dépérissement sont vertes et fonctionnelles (Figure 2D). Dans d'autres, toute la tige dépérit et de nouveaux rejets émergent à partir de la base des souches (Figure 2E). Des pieds de teck présentant un caractère asymptomatique et apparemment sains ont une écorce qui ne présente aucun signe de pourriture ou de brunissement. Cependant, un écorçage partiel permet d'observer une atteinte interne du bois de cœur avec une décoloration brun-noirâtre qui atteint progressivement la partie souscorticale ou phloème (Figure 2F).

\section{Symptômes d'attaque souterraine}

L'observation de la partie souterraine des rejets révèle une attaque au niveau du pivot central. L'attaque peut, dans certains cas, toucher les racines latérales, mais reste limitée. Les racines en dépérissement sont décolorées au niveau du cylindre central avec une coloration brun-noirâtre (Figure 3A). Des pieds présentent également un caractère asymptomatique et apparemment sain. L'écorce, dans ce cas, ne présente aucun signe visible de pourriture ou de brunissement. Néanmoins, un écorçage partiel (ou total) de la racine ainsi qu'une coupe réalisée transversalement ont permis de constater les dégâts (Figures 3B et 3C).

\section{Ravageurs de rejets de souche}

Trois types de ravageurs s'attaquent aux rejets de teck. Il s'agit des termites avec le 
genre Ancistrotermes (Figure 4A); de criquets puants avec l'espèce Zonocerus variegatus (Figure 4B) et de charançons avec l'espèce Xyleborus aquilus (Figures 4C1, 4C2 et 4C3). Les termites (Ancistrotermes) et le charançon (Xyleborus aquilus) s'attaquent aux tiges dans des proportions respectives de 0,83 et $0,62 \%$. $\mathrm{Au}$ niveau des attaques du genre Ancistrotermes, on enregistre des rejets vivants et morts alors qu'au niveau de Xyleborus aquilus, les arbres sont, soit vivants, soit dépéris. Zonocerus variegatus quant à lui, attaque uniquement les feuilles. La proportion de rejets atteinte dans ce cas, est de $3,33 \%$ (Tableau 1).

\section{Modalités d'attaques des ravageurs}

Le genre Ancistrotermes fait des placages de terre argileuse le long de la tige saine ou en dépérissement. En dessous, les termites dévorent partiellement l'écorce (Figure 5A). Dans le cas des rejets morts ou en dépérissement, les termites perforent la zone sous-corticale en créant des galeries d'où sont stockées les terres argileuses digérées qui s'assèchent sous l'effet du soleil en créant des zones de craquement au niveau de l'écorce. Des galeries de termites prolifèrent au niveau de la zone interne du bois et provoquent le dépérissement ou la mort des rejets de souches (Figure 5B). Les criquets puants de l'espèce Zonocerus variegatus s'attaquent préférentiellement aux feuilles basales âgées. Les autres organes ne sont pas attaqués. La consommation a lieu de l'extrémité vers la base des feuilles en procédant par perforation (Figures 6A et 6B). Le criquet ne consomme pas les nervures des feuilles (Figure 6C). Les perforations des feuilles n'entraînent pas la mort des rejets de souches. Xyleborus aquilus s'attaque uniquement aux tiges de teck. Il crée des zones d'entrance ou des galeries dans les tissus qui secrètent de la sciure de bois (Figure 7A). La partie de la tige attaquée devient non fonctionnelle et s'assèche. On assiste à un début de dépérissement par la coloration brune du bois du cœur (Figures 7B).

\section{Champignons isolés}

A l'observation visuelle, les thalles des champignons présentent différentes colorations caractéristiques en fonction des genres. A l'observation microscopique, le mycélium des genres est cloisonné ou non cloisonné, ramifié ou non ramifié et porte des conidies ou non (Figures 8, 9 et 10).

La purification des différents champignons obtenus a permis d'obtenir 9 isolats appartenant à 6 genres dont 5 sont identifiés aux genres, Fusarium, Penicillium, Phellinus, Aspergillus, Trichoderma. Le sixième genre n'a pu être identifié par manque de fructification. Six isolats (isolat A, isolat B, isolat $D$, isolat $G$, isolat $H$ et isolat $I$ ) sont obtenus à partir de l'écorce. Trois isolats (isolat $\mathrm{C}$, isolat $\mathrm{E}$, isolat $\mathrm{F}$ ) sont obtenus au niveau du cylindre central.

Les isolats $\mathrm{A}, \mathrm{B}$ et $\mathrm{C}$ observés appartiennent au genre Fusarium (Figure 8). L'isolat B est identifiée comme Fusarium verticilioides ou Fusarium moniliforme (Figure 8B2). Les isolats D et E appartiennent au genre Penicillium (Figures 9D2 et 9E2). L'isolat $F$ est inconnu de la clé d'identification que nous avons utilisée (Figure 9F2). L'isolat $\mathrm{G}$ est identifié comme Phellinus noxius (Figure 10G2) ; l'isolat $\mathrm{H}$ appartient au genre Aspergillus (Figure 10H2). Enfin, l'isolat I appartient au genre Trichoderma (Figure 10I2). 


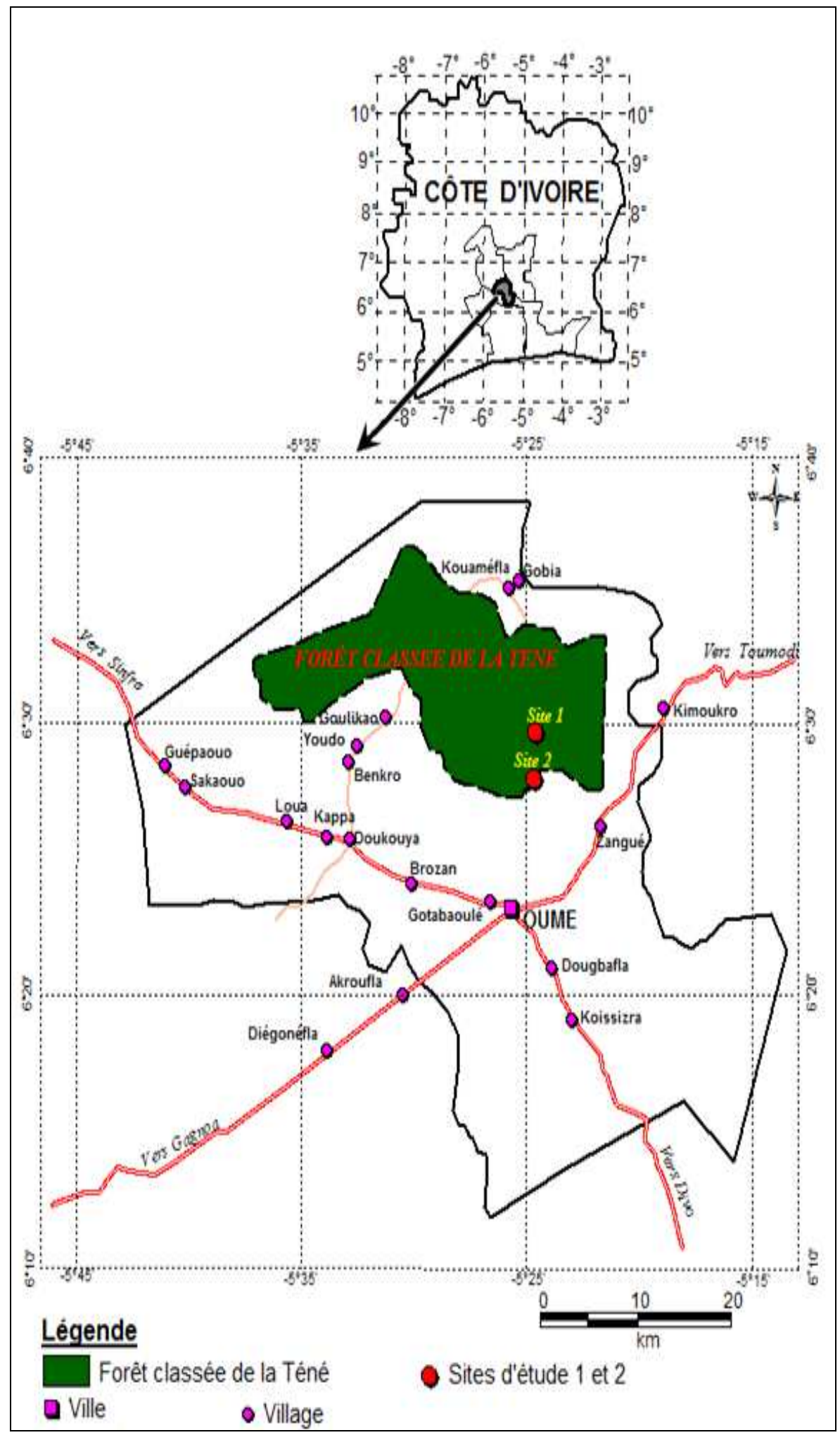

Figure 1: Localisation des sites d'étude dans la forêt classée de la Téné (Source : CCT, 2013). 

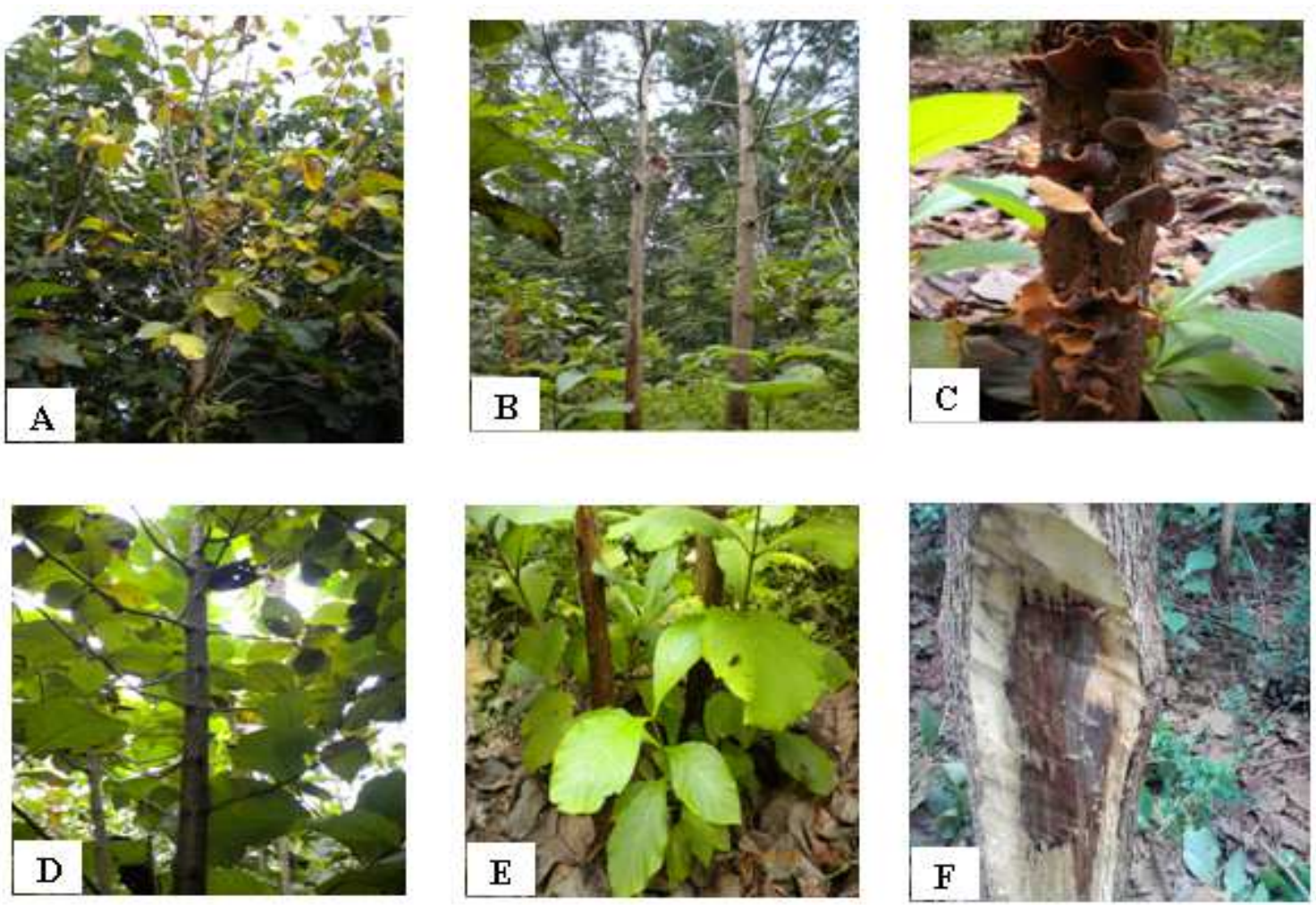

Figure 2: Rejets de teck présentant des symptômes de dépérissement. A : Rejets de teck en début de dépérissement avec une défoliation apico-basale ; B : Rejets de teck complètement dépéris; C : Tige symptomatique envahie par des champignons saprophytes; D: Tige de teck en début apparent de symptômes avec feuilles médianes assimilatrices ; E : Rejets émergés à la base d'anciens rejets de souche complètement dépéris ; F : Galerie dans un tronc de teck avec une décoloration brune du bois de cœur.
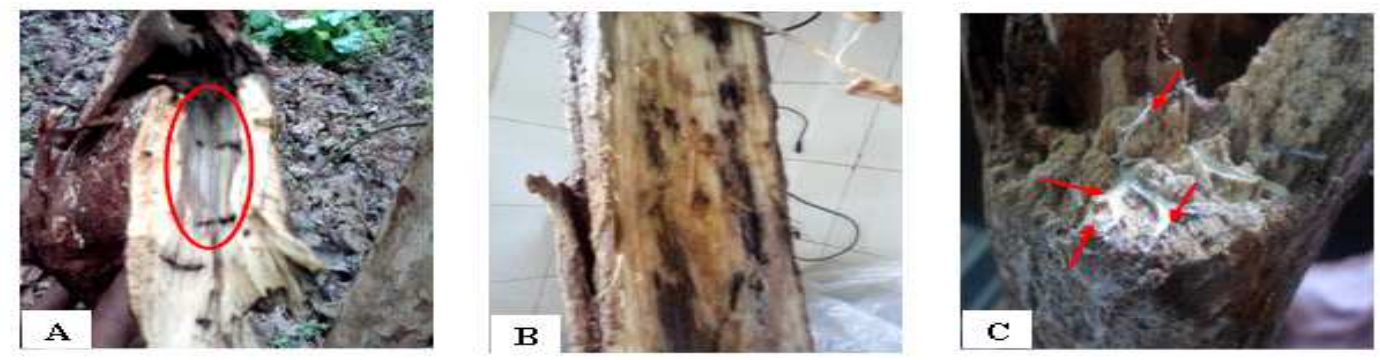

Figure 3: Racines de teck présentant des symptômes de dépérissement. A : Partie d'un pivot complètement dépéri présentant un cylindre central décoloré ; B : Portion d'un pivot écorcée partiellement montrant une coloration brune interne ; $\mathrm{C}$ Section transversale d'un pivot présentant des tissus remplis de fructifications fongiques. 

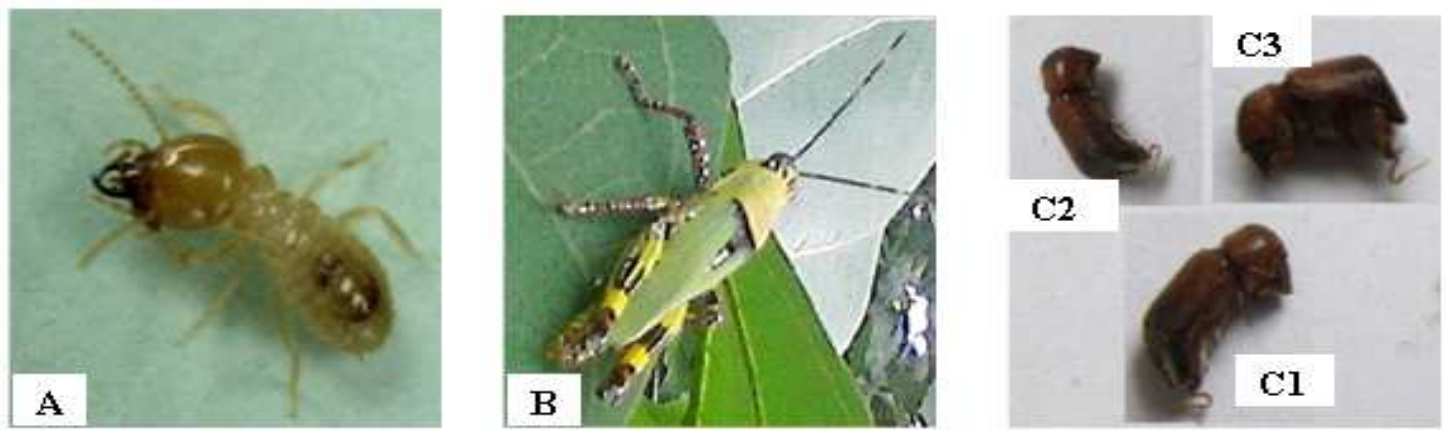

Figure 4: Quelques insectes parasites des rejets de souche de teck. A : genre Ancistrotermes (G x16) ; B: Zonocerus variegatus (G x 5); C1, C2 et C3 : Xyleborus aquilus (G x 10).
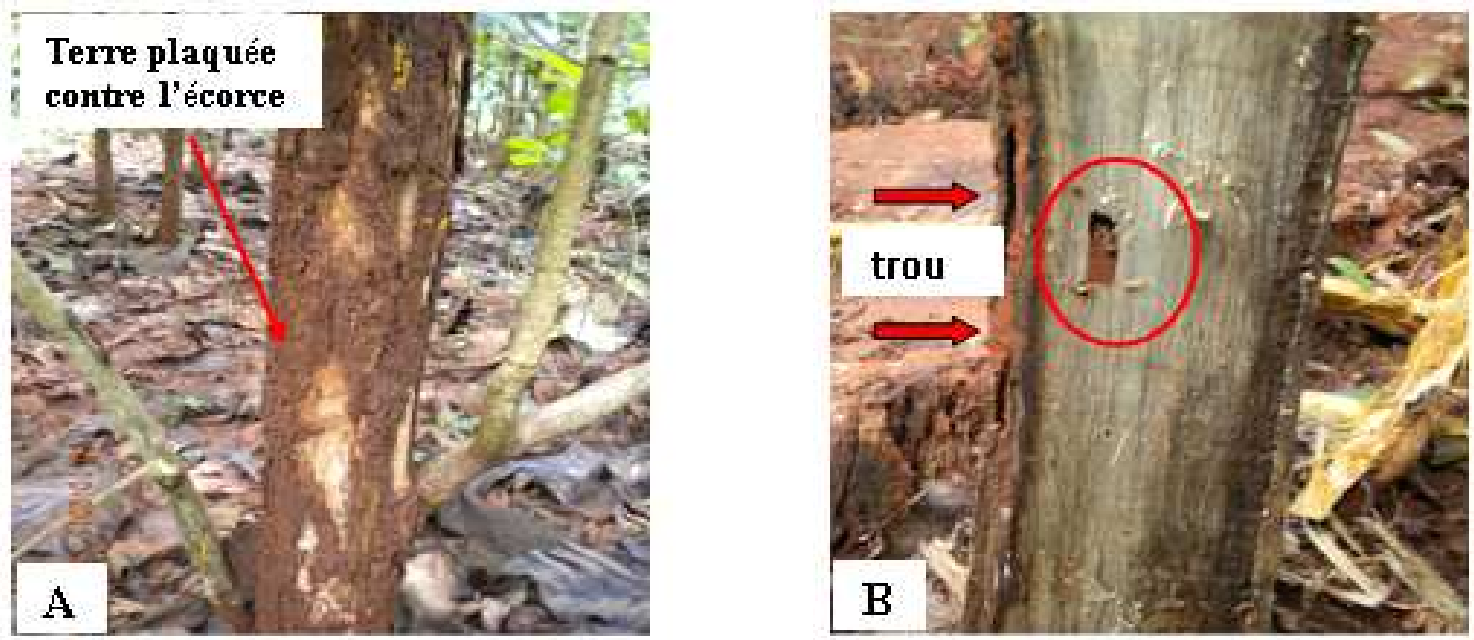

Figure 5: Attaques par Ancistrotermes de tige de teck avec placage de terre sur l'écorce. A : placage de terre contre l'écorce d'un rejet vivant de teck par $g$. Ancistrotermes; B : Trous d'entrance ou d'émergence du $g$. Ancistrotermes avec bourrage d'argile dans la zone corticale et sous-corticale suivi du décollement de l'écorce sous l'effet de soleil. 

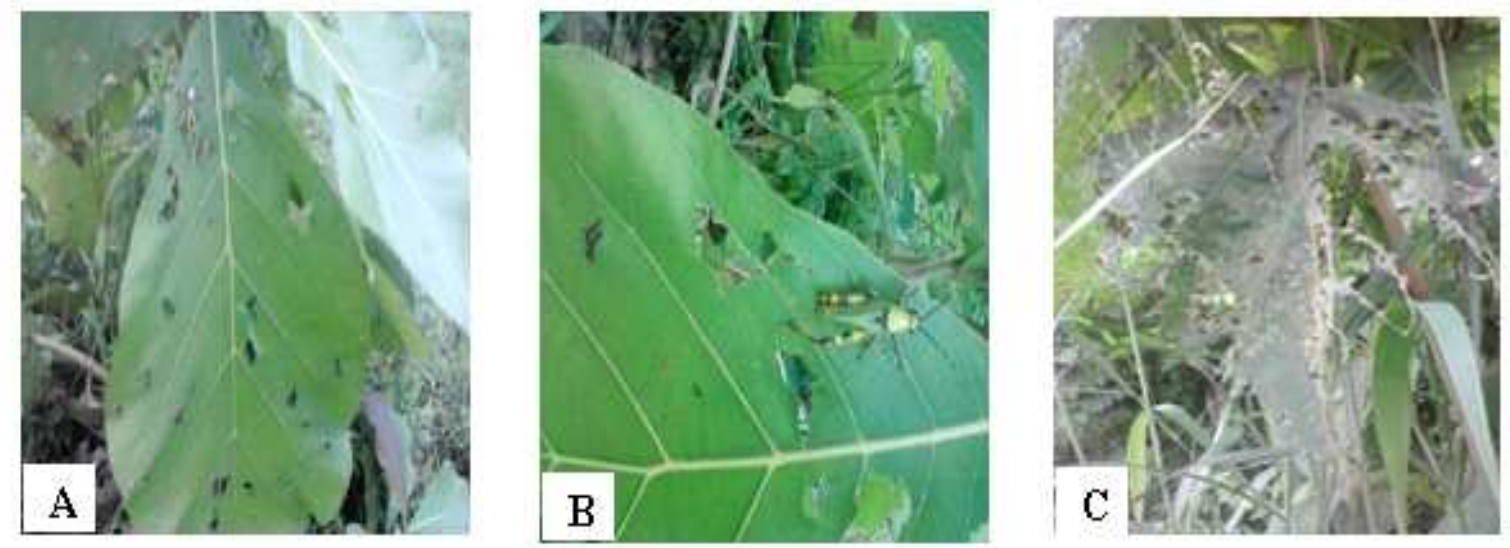

Figure 6 : Attaque de feuilles de teck par Zonocerus variegatus. A et B : Début d'attaque par perforation du limbe de teck ; $\mathrm{C}$ : Attaque totale avec déchiquètement d'une feuille de teck.
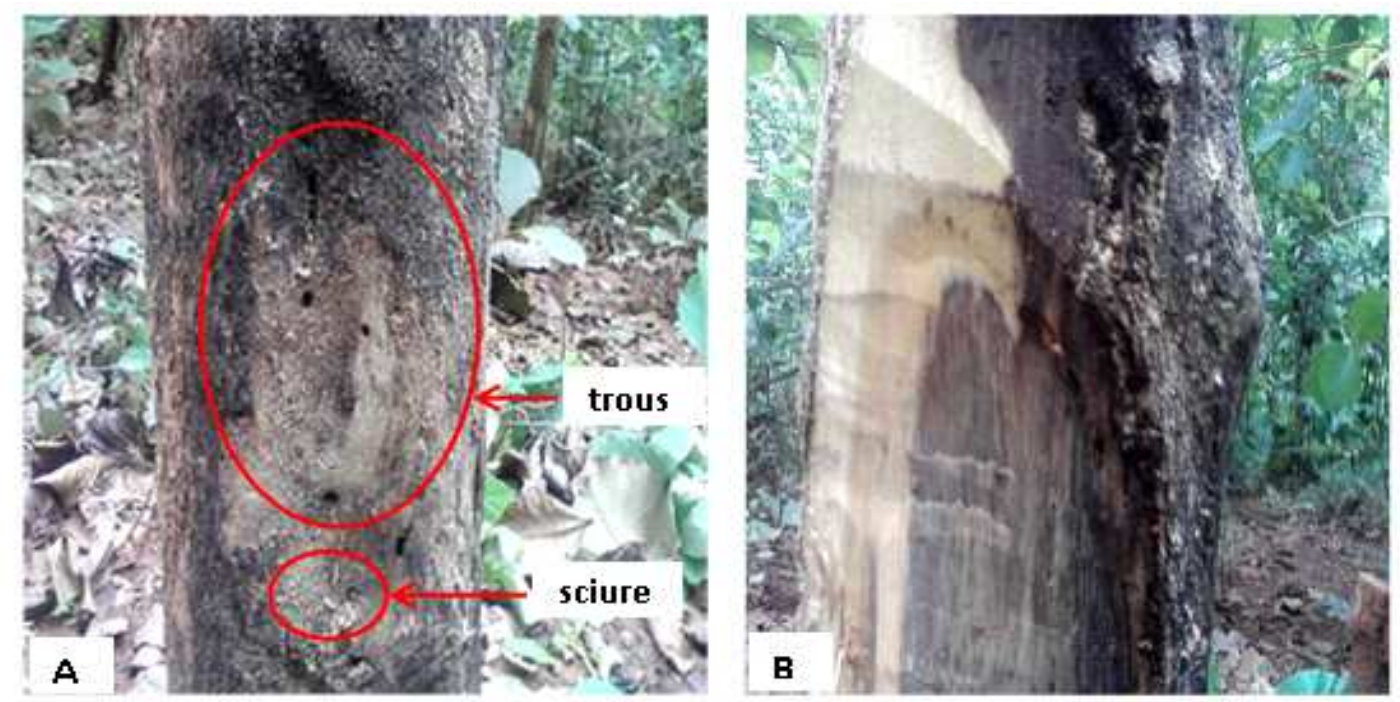

Figure 7: Rejets de teck attaqués par des coléoptères. A : Trous d'entrance et d'émergence de Xyleborus aquilus sur un rejet de teck en début de dépérissement; présence de sciure à proximité des trous ; B : Ecorçage partiel de tiges de teck montrant une écorce encore vivante et un bois de cœur brun. 

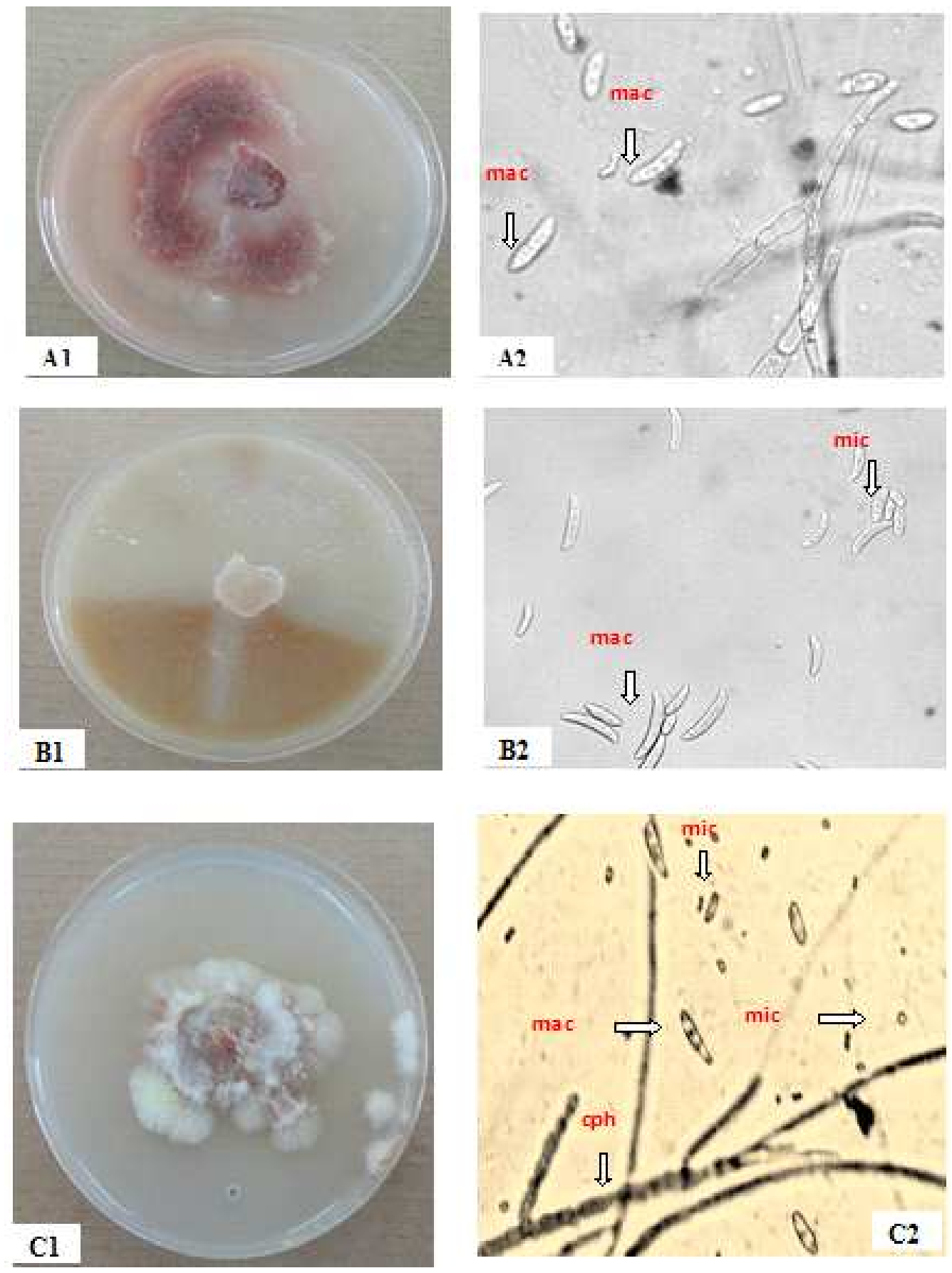

Figure 8: Vues macroscopique (A1, B1, C1) et microscopique (A2, B2, C2) des genres Fusarium isolés à partir des pivots de teck. A2-Fusarim sp. (Gx100) isolé à partir de l'écorce; B2-Fusarium verticilioides (Gx100) isolé à partir de l'écorce ; C2-Fusarium sp. (Gx100) isolé à partir du cylindre central avec mic-microconidie ; macmacroconidie et cph-conidiophore. 

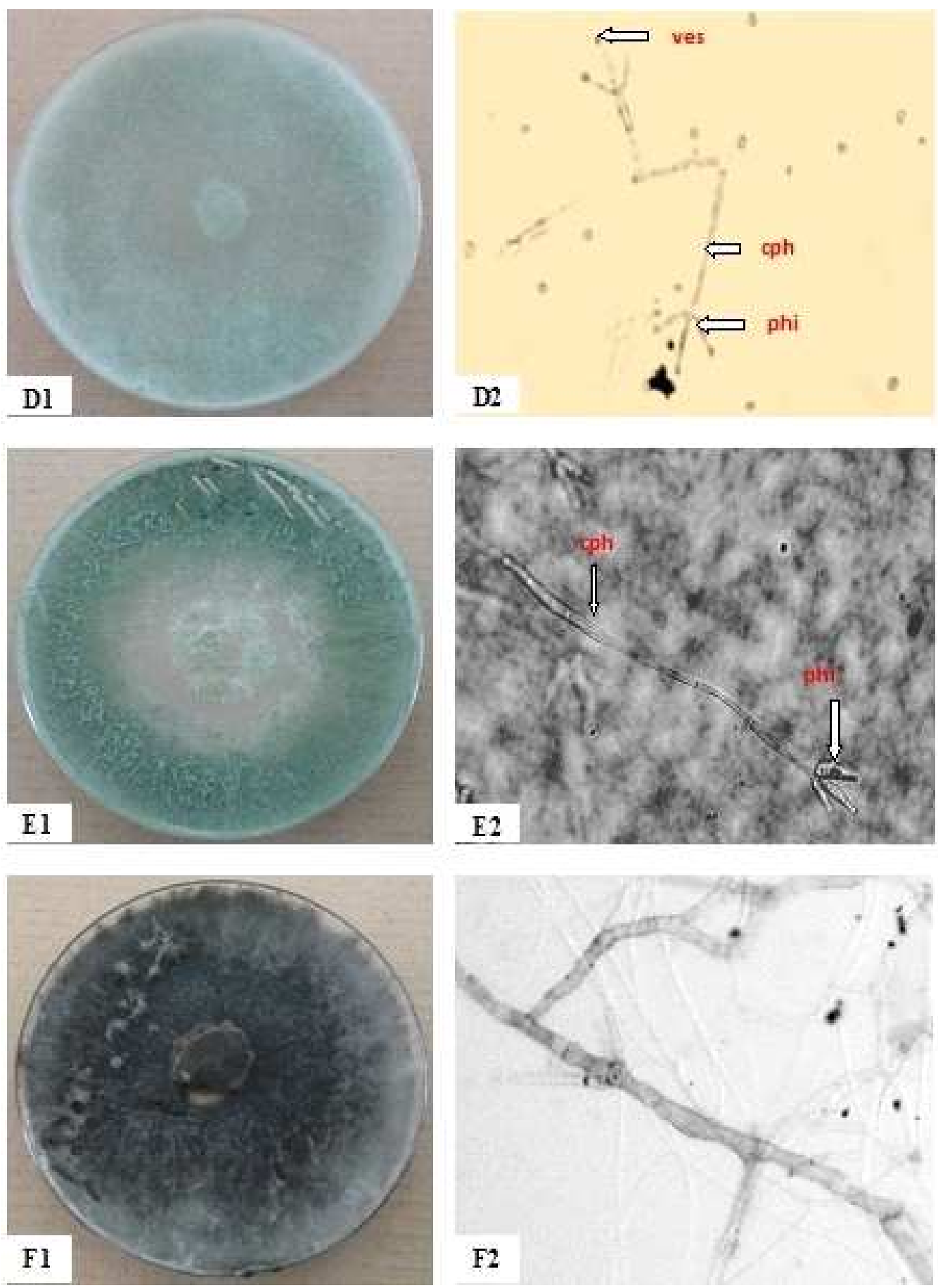

Figure 9: Vues macroscopique (D1, E1, F1) et microscopique (D2, E2, F2) des genres Penicillium et une souche inconnue isolés à partir des pivots de teck. D2-Penicillium sp. (Gx20) isolé à partir de l'écorce ; E2-Penicillium sp. (Gx20) isolé à partir du cylindre central ; F2-Souche inconnue (Gx100) isolée à partir du cylindre central avec ves-vésicule ; cph-conidiophore et phi-phialide. 

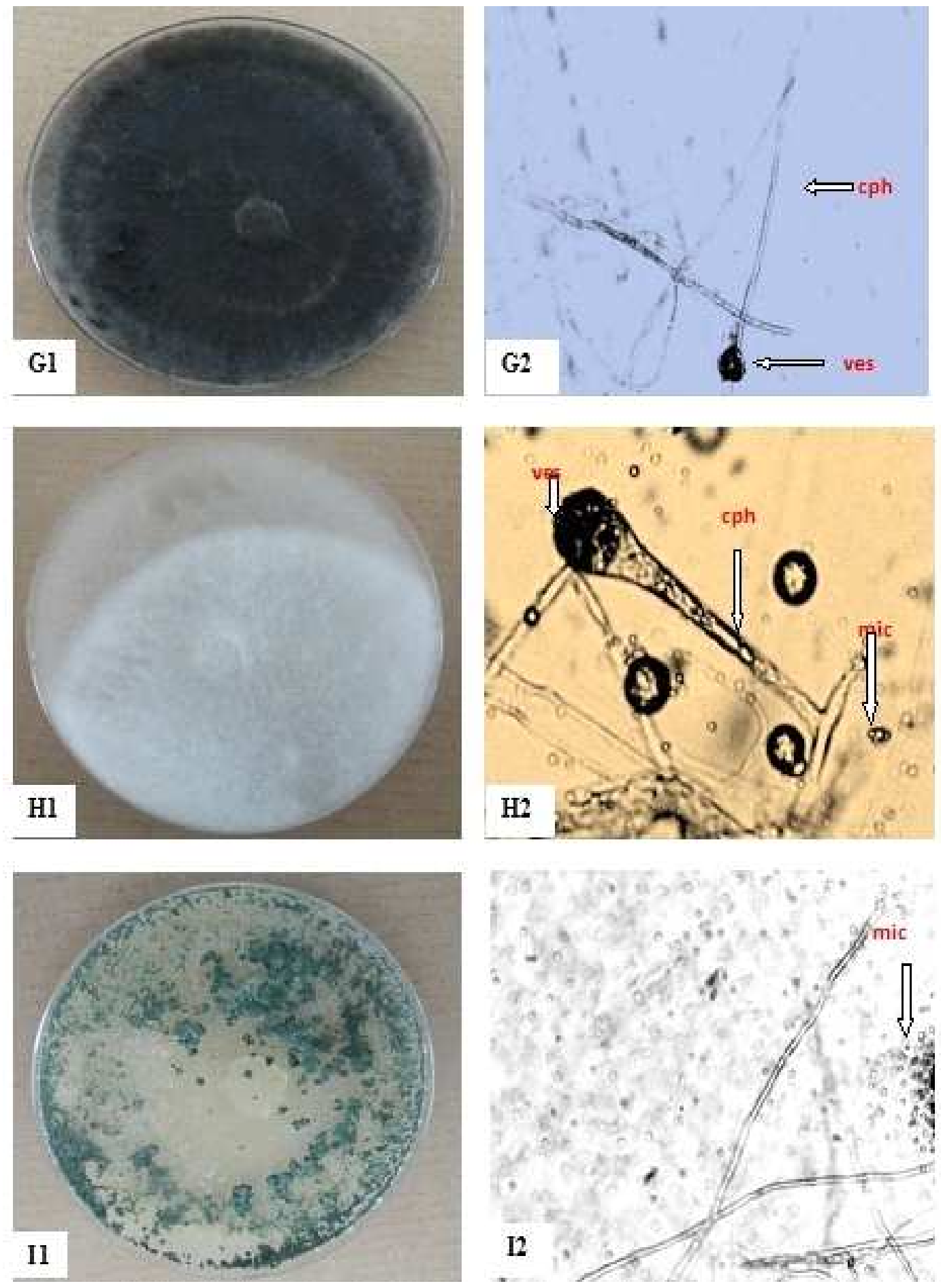

Figure 10: Vues macroscopique (G1, H1, I1) et microscopique (G2, H2, I2) des genres Phellinus, Aspergillus et Trichoderma isolés à partir des pivots de teck. G2- Phellinus noxius (Gx100) isolé à partir de l'écorce ; H2- Aspergillus sp. (Gx100) isolé à partir de l'écorce ; I2- Trichoderma sp. (Gx100) isolé à partir de l'écorce avec mic-microconidie et ves-vésicule. 
Tableau 1 : Parasites et proportion de rejets attaqués.

\begin{tabular}{|c|c|c|c|c|}
\hline Type de ravageurs & $\begin{array}{l}\text { Nombre de } \\
\text { rejets } \\
\text { attaqués }\end{array}$ & $\begin{array}{l}\text { Organes } \\
\text { attaqués }\end{array}$ & Etat sanitaire & $\begin{array}{c}\text { Taux de rejets } \\
\text { par rapport a } \\
\text { la densité } \\
\text { initiale }(\%)\end{array}$ \\
\hline $\begin{array}{c}\text { g. Ancistrotemes } \\
\text { (termites) }\end{array}$ & 4 & $\begin{array}{c}\text { tiges } \\
\text { (écorce) }\end{array}$ & vivant/mort & 0,83 \\
\hline $\begin{array}{c}\text { Zonocenus variegatus } \\
\text { (criquets puants) }\end{array}$ & 16 & feuilles & vivant & 3,33 \\
\hline $\begin{array}{c}\text { Xylebons aquilus } \\
\text { (charançons) }\end{array}$ & 3 & tiges & vivantdépérissement & 0,62 \\
\hline TOTAL & 23 & - & - & 4,78 \\
\hline
\end{tabular}

\section{DISCUSSION}

Cette étude a montré la présence de champignons pathogènes dans le sol forestier de Téné. Elle confirme d'une part, les travaux de Kouadio et al. (2008) sur le dépérissement des essences de plantation en zone forestière semi-décidue et d'autre part ceux de Zézé et al. (2007) dans l'étude des champignons endomycorhizogènes dans ladite zone. Les prélèvements réalisés sur les pivots racinaires montrent que le dépérissement est associé à des pathologies biologiques de type fongique. Neuf isolats fongiques appartenant à six genres ont été isolées : Fusarium, Penicillium, Phellinus, Aspergillus, Trichoderma et un sixième genre non identifié par manque de fructification. L'écorce, couche superficielle externe est plus victime des attaques fongiques comparée au cylindre central. En effet, des neuf isolats identifiés, six ont été obtenus à partir de l'écorce (Fusarium sp., Fusarium verticilioides, Penicillium sp, Phellinus noxius, Aspergillus sp., Trichoderma sp.). Par contre, trois seulement étendent leur agressivité jusqu'au cylindre central (Fusarium sp., Penicillium sp., souche inconnue). Ces derniers, probablement les premiers à coloniser le milieu, étendent rapidement leur agression jusqu'au cylindre central. Ces mêmes résultats ont été rapportés par Gnancadja et al. (2004) qui ont également noté que le premier champignon à coloniser l'hôte prend la position dominante et les espèces mineures ont une courte durée de vie. Ces champignons, appelés trachéomycoses, agissent, soit par action des toxines libérées, soit par obstruction du cylindre central, empêchant ainsi la circulation de la sève brute par le xylème.

La pourriture brune observée sur les pivots de rejets est associée à la présence de Phellinus noxius. Des résultats similaires ont également été rapportés par Kouadio et al. (2008) au cours de leurs travaux réalisés à Oumé et Bouaflé, deux zones semi-décidues de Côte d'Ivoire. Selon ces auteurs, la coloration brune observée sur les racines est le fait d'agents de pourridiés, notamment ceux de Phellinuss noxius qui occasionnent une décoloration et une nécrose qui sont consécutives à une occlusion des vaisseaux conducteurs par envahissement progressif du champignon. Selon les racines touchées, le développement symptomatique chez l'hôte peut être plus ou moins rapide. Les organes foliaires jeunes en croissance active sont les plus touchés car ils constituent le puits essentiel des nutriments minéraux (sève brute). Par conséquent, ils sont les premiers à exprimer les symptômes. Ailleurs, en Malaisie, les travaux de Bhat et al. (2005), Mohd Farid et al. (2001 et 2006) et ceux de N'Guessan et al. (2012) réalisés à Irobo et Bouaflé (Côte d'Ivoire) ont indexé ce 
champignon comme agent pathogène causant la pourriture brune aussi bien chez le teck que chez l'hévéa. Ils signalent que ces pourritures brunes sont caractérisées par la dégradation préférentielle des polysaccharides pariétaux. Aussi, le traumatisme physique occasionné par le parasite du système racinaire des rejets attaqués ainsi que les perturbations métaboliques qu'ils entraînent sont à l'origine des manifestations symptomatiques de la maladie au niveau du feuillage (jaunissement, chute prématurée et progressive du feuillage), de la tige (fendillement de l'écorce qui se détache du tronc) et du système racinaire.

Les souches de Aspergillus et de Penicillium, isolées au niveau l'écorce sont des champignons saprophytes apparaîssant comme des ennemis de faiblesse et de stress d'une infection préalablement établie et dont le rôle serait d'aggraver la maladie. Agrios (2005) indique à ce sujet que les arbres sous stress sont beaucoup sujets aux infections pathogènes. Par ailleurs, l'activité de ces deux champignons pourrait être favorisée par celles des coléoptères qui font des perforations et des galeries pour pondre leurs œufs. En effet, ces souches endommagent le cambium vasculaire et stimulent aussi le dépérissement, ce qui a pour conséquence la fissuration longitudinale de l'écorce et la destruction des éléments du xylème et $\mathrm{du}$ phloème fonctionnels. Aussi, Vansteenkiste et al. (2005) signalent que les larves, colonisant l'écorce, réduiraient ou empêcheraient la circulation de la sève élaborée depuis les feuilles jusqu'aux racines créant alors un stress au niveau de la plante. Les conclusions sont également partagées par Kouadio et al. (2008) sur Tectona grandis et N'guessan (2009) sur Terminalia ivoriensis.

Dans d'autres circonstances, les genres Aspergillus, Penicillium et Trichoderma sont utilisés comme agent de lutte biologique. Leur action consiste, d'une part, en une augmentation de la densité de propagules et d'autre part, en une production de substances diffusibles et toxiques dans le sol. Ces substances sécrétées entravent le développement de certains champignons reconnus comme pathogènes sur les essences forestières, notamment Fusarium, Diplodia, et Verticillium. Mohd Farid et al. (2009) expliquent cela par une activation du système de défense de la plante, une augmentation de l'activité chitinase et peroxidase et un accroissement de l'activité enzymatique dans les feuilles induisant ainsi une résistance systémique chez ces plants. Dans nos travaux, les effets des genres Aspergillus, Penicillium et Trichoderma se seraient avérés inefficaces contre les souches de Phellinus noxius. Ces derniers, probablement en surnombre, les auraient dominés au point de provoquer le dépérissement, puis la mort des rejets de souches. Le genre Fusarium, par contre, est un parasite qui colonise les racines des plantes attaquées en obstruant les canaux (N'guessan, 2009). Enfin, la présence de Trichoderma sp. est d'une importance capitale du fait qu'il est un redoutable antagoniste des champignons phytopathogènes (Afouda et al., 2013). Cela constitue des pistes pour une lutte microbiologique contre certains des agents pathogènes que nous avons identifiés.

La proportion de rejets attaqués par les insectes ravageurs $(4,78 \%)$ reste faible comparée au taux de mortalité de l'ensemble du peuplement régénéré (24\%). La différence pourrait être imputable à d'autres facteurs, notamment de gestion sylvicole (densité), au sol, au stress ou au déficit hydrique. Le faible taux d'attaque par les ravageurs pourrait être lié, au bon entretien des parcelles. Nos résultats sont similaires à ceux obtenus par plusieurs auteurs, notamment Kouadio et al. (2008), Gotoh et al. (2003) et Mohammad et Sunami (2004). Ces auteurs ont constaté un faible taux d'attaque du teck par les ravageurs avec une proportion d'environ 2,2\% Ils en sont arrivés à conclure que le dépérissement 
du teck n'était pas le fait des ravageurs, mais qu'ils pouvaient être des facteurs de stress suffisants dont les effets cumulatifs fragiliseraient le teck.

Trois types de ravageurs ont été identifiés au cours des travaux réalisés. Il s'agit des termites du genre Ancistrotermes, des criquets puants dont l'espèce Zonocerus variegatus et du coléoptère Xyleborus aquilus.

Les peuplements de rejets de Tectona grandis sont victimes des attaques des termites du genre Ancistrotermes. Cette présence des termites est liée aux conditions favorables qu'offre ce milieu notamment, humidité et débris végétaux issus d'éclaircies à partir desquels ils construisent leurs nids. De pareils résultats ont été obtenus par Maldague (2003) qui signale que les nids constituent un milieu favorable au développement des champignons dont les mycéliums prospèrent de la meule et sa surface où ils forment un mince feutrage, le velours. Le genre Ancistrotermes que nous avons observé s'attaque aussi bien aux rejets vivants qu'aux rejets morts ou en dépérissement. A cet effet, Tra Bi (2013) note que le régime alimentaire de Ancistrotermes est essentiellement constitué de feuilles et de bois morts qu'ils prédigèrent sous forme de meule sur laquelle se développera le champignon du genre Termitomyces. Nos résultats ont également montré la présence de galerie d'argile le long des rejets ainsi qu'au niveau du sol racinaire, répondant ainsi à un besoin vital. Ces résultats sont en accord avec ceux obtenus par Anani Kotoklo et al. (2010) lors de l'inventaire des termites des arbres sur le campus de l'Université de Lomé (Togo). Ils stipulent que les attaques sont localisées au niveau de l'écorce, des troncs et des branches avec souvent de spectaculaires constructions de galeries et des placages argileuses qui entravent la circulation de la sève. Apkesse et al. (2008) soulignent que ces placages externes sur la tige servent de passage pour attaquer le végétal de l'extérieur vers l'intérieur en consommant successivement le cortex externe, le parenchyme et le cambium. La création de placage répond à un besoin vital (nourriture) et de protection contre les rayons ultraviolets du soleil et surtout contre les attaques de fourmis. En effet, les termites plaquent la nourriture à consommer contre l'écorce et entrent en dessous en procédant à de petites perforations. Une fois sous l'écorce, Ancistrotermes accumule de quantités importantes de terre argileuse. Cette accumulation conduit au craquement et au décapage de l'écorce sous l'effet de gonflement d'argile. Ailleurs, au Benin, les populations de termites se retrouvent à la fois sur le sol et sur les pieds de teck. Ancistrotermes est un ravageur potentiel pour le teck (Gbenyedji et al., 2011). La proportion de rejets attaqués par Zonocerus variegatus $(3,33 \%)$ est la plus importante comparée à celle du genre Ancistrotermes (0,83\%) et de Xyleborus aquilus (0,62\%). Malgré cette importance, ce taux reste marginal par rapport à d'autres cultures infestées en Afrique de l'Ouest. En effet, au Nigeria, 50\% des cultures de manioc sont détruites (Kekeunou et al., 2014) tandis qu'au Benin, $30 \%$ de pertes en maïs sont signalées (Balogun et al., 2004). En outre, lors de l'inventaire, nous n'avons pas observé de cas de mortalité liée aux attaques des criquets. Leurs attaques sont saisonnières, elles interviennent au début et à la fin de la saison des pluies, probablement à cause d'une nourriture plus abondante à cette période. Ces résultats sont confirmés par les travaux de James et al. (2000) et Messi et al. (2006) qui signalent que les attaques de Zonocerus variegatus sont plus graves en saison pluvieuse qu'en saison sèche. Par ailleurs, au cours de nos inventaires de terrain, nous avons observé la présence de Zonocerus variegatus dans la végétation environnante (Talinum triangulare, Aspilla lacifolis, Aspilla africana, Tridax esculenta, Chromolaena odorata) mais 
également dans les champs de Manihot esculenta. Les feuilles de ces espèces contribuent a la croissance de Zonocerus variegatus (Okonji et al., 2013) Cela démontre le caractère polyphage du criquet puant. Des études réalisées par James et al. (2015) ont montré que Zonocerus variegatus était attiré par différentes plantes contenants des alcaloïdes de pyrrolicidines dont le criquet s'en sert pour se protéger chimiquement ainsi que ses œufs contre les parasites. Zonocerus variegatus a donc un comportement pharmacophage par rapport aux plantes attaquées. Cependant, Onzo et al. (2014) notent l'absence d'alcaloides dans la composition chimique des feuilles de teck. Les attaques se font soit en masse ou de manière solitaire par des perforations du limbe foliaire, entraînant une chute prématurée. Si des cas de mortalité n'ont pas pu être observées lors des essais, il n'est pas exclu qu'une défeuillaison intense n'entraîne la suppression de montée de sève brute et de photosynthétats. Ce phénomène provoque alors un stress hydrique et alimentaire en composés carbonés.

L'attaque par Xyleborus aquilus a lieu uniquement au niveau de la tige principale. L'attaque des rejets de teck par ce coléoptère reste très limitée $(0,62 \%$ de tiges attaquées). Ce coléoptère marque sa présence par des trous d'entrance ou galeries. L'émission de la sciure de bois ainsi que la décoloration brune observée sur les tiges est le résultat d'une désagrégation fonctionnelle et structurale des tissus du xylème et du phloème. L'on pourrait assister dans ce cas, à un arrêt du transport des substances minérales et de synthèse. De pareilles conclusions sont aussi rapportées par Anonyme (2012). Il note que les galeries sous corticales et transversales perturbent la circulation de la sève, déprécient la qualité et produisent une sensibilité aux dégâts de vents. Ces morsures de nutrition peuvent provoquer des ruptures de pousses et entraîner des fourchaison sur les rejets.

\section{Conclusion}

Les résultats montrent que des pathologies dues aux ravageurs et aux champignons sont associées au dépérissement des rejets de teck. Parmi les ravageurs, des termites du genre Ancistrotermes, des criquets dont l'espèce Zonocerus variegatus et un coléoptère, Xyleborus aquilus sont recensés. Ces ravageurs n'ont pu être identifiés comme agents de dépérissement du teck. En revanche, ils sont présentés comme des ennemis de faiblesse d'un phénomène préalablement établi et dont les actions fragiliseraient l'intégrité biologique et physique des rejets. Cependant, le genre Ancistrotermes, provoque chez le teck le décapage et le craquement de l'écorce. La construction de galeries dans les structures internes $\mathrm{du}$ bois et $\mathrm{du}$ phloème provoque parfois le dépérissement puis la mort des rejets. La pourriture brune associée au dépérissement a révélé la présence de six agents pathogènes de type fongique: $g$. Fusarium, g. penicillium, Phellinus noxius, $g$. Aspergillus, g. Trichoderma et un genre inconnu par faute de sporulation. Parmi ces agents pathogènes, Phellinus noxius est reconnu comme associé à la pourriture brune racinaire et provoquant le dépérissement puis la mort du teck.

\section{CONFLIT D'INTERET}

Les auteurs déclarent qu'il n'existe aucun conflit d'intérêt dans cet article.

\section{CONTRIBUTIONS DES AUTEURS}

BNBVB était concepteur et investigateur principal. Il a effectué les travaux de terrain et les tests de laboratoire. $\mathrm{K}$ AN'G et KFJMK ont supervisé le travail. FA TB a assisté financièrement le travail. KK a coordonné l'ensemble des travaux de 
recherche. Tous ces auteurs ont participé à la rédaction du manuscrit.

\section{REMERCIEMENTS}

La présente étude a été réalisée grâce à une subvention spécifique du Centre de Gestion de Gagnoa de la Société de Développement des Forêts (SODEFOR) et à l'apport technique du Laboratoire de Phytopathologie de l'Université Félix Houphouët-Boigny. Nous leur témoignons notre profonde gratitude.

\section{REFERENCES}

Anani Kotoklo E, Kasseney DB, Nyamador W, Ketoh KG, Glitho AI. 2013. Attaques des arbres par les termites sur le campus de l'Université de Lomé (Togo). International Journal of Biological and Chemical Sciences, 4(1): 61-68.

Anonyme. 2012. Le charançon de la patience. Ministère de l'agriculture, de l'agroalimentaire et de la forêt (France). Département de la santé des forêts, p. 2.

Afouda LCA, Zinzou V, Balogoun RK, Onzon A, Ahohuendo BC. 2013. Inventaire des agents pathogènes de l'anacardier (Anacardium occidentale L.) au Benin. Bulletin de Recherche Agronomique du Benin, 73 : 13-19.

Agrios GN. 2005. Plant Pathology ( $5^{\text {th }}$ edn). Elsevier Academic Press of San Diego: San Diego.

Akpesse AA, Kouassi P, Tano Y, Lepage M. 2008. Impact des termites dans les champs paysans de riz et de maïs en savane sub-soudanienne (Booro-Borotou, Côte-d'Ivoire). Sciences et Nature, 5(2): 121-131.

Balogun SA, Fagade OE. 2004. Entomopathogenic fungi in population of Zonocerus variegatus (L.) in Ibadan, Southwest, Nigeria. African Journal of Biotechnology, 3(8): 382-386.
Barnett HL, Barry BH. 1960. Illustration genera of imperfect fungi. Burgess Publishing Company, 3: 12-160.

Gbenyedji KBJN, Anani Kotoklo E, Amevoin K, Glitho AI. 2011. Diversité spécifique des termites (Isoptera) dans deux plantations de tecks (Tectona grandis L.) au sud du Togo. International Journal of Biological and Chemical Sciences, 5(2): 755-765.

Bhat KM, Thulasidas PK, Florence EJM. 2005. Wood durability of home-garden teak against brown-rot and white-rot. Kerala Forest Research Institute, 19(6): 654-660.

CCT (Centre de Cartographie et de Télédétection). 2013. Centre de Cartographie et de Télédétection. In : Tra Bi CS. 2013. Diversité spécifique et dégâts des termites dans les cacaoyères (Theobroma cacao L., 1753) de la région d'Oumé en côte d'Ivoire. Thèse de Doctorat, Université Félix HouphouëtBoigny, Côte d'Ivoire, p. 252.

CNRA. 2013. Rapport annuel des activités de recherche. Centre National de Recherche Agronomique (Côte d'Ivoire), p. 30.

FAO. 2007. FOSA Document National de Prospective Côte d'Ivoire. Département des Forêts. Archives de Documents de la $F A O$. FAO : Rome, Italie.

Gnancadja A, Ouazzani T, Badoc A, Douira A. 2004. Test de détection des contaminants fongiques des grains de riz en fin de cycle végétatif. Bull. Soc. Pharm., 143: 39-50.

Gotoh T, Kotulai JR, Matsumoto K. 2003. Stem borers of teak and yemane in Sabah (Malaysia), with analysis of attacks by teak beehole borer (Xyleutes ceramica Walker). Japan Agricultural Research Quarterly, 37(4): 253-261.

James A, Timbilla, Yeboah-Gyan K, Lawson BWL, Hartmann T. 2015. A Grasshopper and its beneficial drug source: the African 
Story of Zonocerus variegatus and the Neophyte Chromolaena odorata. Research Journal of Phytochemistry, 9: 1-15.

James B, Yaninek J, Neuenschwander P, Cudjoe A, Modder W, Echendu N, Toko M. 2000. Lutte contre les ravageurs du manioc. Guide de la pratique de lute intégrée à l'usage des vulgarisateurs, archives de documents de la FAO, p. 21.

Kouadio NV, Amonkou YE, Aké S. 2008. Lutte contre le dépérissement des essences de plantations. Cas de Terminalia Spp et de Tectona grandis (teck) en Côte d'Ivoire. Rapport technique SODEFOR/ITTO, p. 87.

Koné KHC. 2012. Etude écologique et phytosociologique des peuplements monospécifiques à haut rendement ligneux de Tectona grandis L. f. (Verbenaceae) dans les forêts classées de la Sangoué et de la Rasso en côte d'ivoire. Thèse de doctorat, Université Félix Houphouët-Boigny, Côte d'Ivoire, p. 221.

Kekeunou S, Yetchom-Fondjo FA, Yana W, Missoup AD, Heumou C, Yengek F, Prombo C. 2014. Life Cycle of Zonocerus variegatus (Orthoptera: Pyrgomorphidae) in the Areas of Equatorial Climate of Cameroon with Uni-and Bimodal Distribution of Rainfall. Academic Journal of Entomology, 7(3): 88-101.

Maldague M. 2003. Etude des termites de la région de Bambesa (RDC) en relation avec la matière organique du sol. Bulletin de l'ANSD, 4: 7-75.

Messi J, Kekeunou S, Weise S. 2006. Abundance and life cycle of Zonocerus variegatus (Orthoptera: Pyrgomorphidae) in the humid forest zone of Southern Cameroon. Entomological Sciences, 9: 24-30.

Mohammad M, Sunami G. 2004. The resistance of 62 indenosian wood species against borer. Journal Penelitian Hasil Hutan, 22(3): 183-191.

Mohd Farid A, Lee SS, Maziah Z, Patahayah M. 2009. Pathogenicity of rigidoporus microporus and phellinus noxius against four major plantation tree species in peninsular Malaysia. Journal of Tropical Forest Science, 21(4): 289-298.

Mohd Farid A, Lee Ss, Maziah Z, Rosli H, Norwati M. 2006. Basal root rot, a new disease of teak (Tectona grandis L.) in Malaysia caused by Phellinus noxius. Malaysian Journal of Microbiology, 1: 40-45.

Mohd Farid A, Maziah Z, Ab Rasip AG, Noraini Sikin Y. 2001. Preliminary study on pathogenicity of three root disease fungi on Azadirachta excelsa (Sentang). Journal of Tropical Forest Science, 13: 554-558.

N'Guessan AC. 2009. Etude de la mycoflore associée au dépérissement de Terminalia ivoriensis, A. Chev. (Combretaceae), Terminalia superba Engel. et Diels (Combretaceae) et Tectona grandis L. f. (Verbenaceae) : cas des forêts classées de Irobo et Bouaflé en Côte d'Ivoire. DEA Agrophysiologie, UFR Biosciences, Université de Cocody-Abidjan, p. 72.

N'Guessan KA, Wahounou PJ, Coulibaly B, Dupuy B. 2012. Bien planter le teck en Côte d'Ivoire. CNRA, Direction des programmes de recherche et de l'appui au développement, p. 4.

Okonji RE, Ehigié OL, Agboola FK. 2013. Isolation and kinetic properties of arginase in the gut of grasshopper (Zonocerus variegatus Linn). International Journal of Biological and Chemical Sciences, 7(1): 1-15.

Onzo FC, Azokpota P, Agbani P, Gbaguidi F, Hounhouigan DJ, Kossou D. 2014. Caractéristiques physico-chimiques, phytochimiques et toxicité des espèces végétales utilisées comme emballages 
alimentaires en Afrique de l'Ouest. International Journal of Biological and Chemical Sciences, 8(4): 1504-1516.

Rapilly F. 1968. Les techniques de mycologie en pathologie végétale. Centre National de Recherche Agronomique. Annales des Epiphytes, 19: 1-100.

Tra Bi CS. 2013. Diversité spécifique et dégâts des termites dans les cacaoyères (Theobroma cacao L., 1753) de la région d'Oumé en côte d'Ivoire. Thèse de Doctorat, Université Félix HouphouëtBoigny, Côte d'Ivoire, p. 252.

Vansteekiste D, Tirry L, Acker VJ, Stevens M. 2005. Predispositions and symptoms of Agrilus borer attack in declining oak trees. Annals of Forest Science, 61: 815823.

Voui Bi BNB, N'guessan KA, Tapé Bi FA, Kamanzi K. 2012. Résultats après un an de traitement en taillis de peuplement de Tectona grandis L. f (teck) en zone semidécidue de Côte d'Ivoire. Journal of Animal and Plant Sciences, 16(2): 23212335.
Voui Bi BNB, N'guessan KA, Koné KHC, Tapé Bi FA, Kamanzi K. 2015. Effets du nombre de rejets par souche et des cultures intercalaires (Arachis hypogaea L., Zea mays L., Phaseolus vulgaris L.) sur la croissance en taillis de Tectona grandis (teck) en zone semi-décidue à Téné, Oumé, Côte d'Ivoire. Afrique Science, 11(6) : 232-249.

Yedmel MSC. 2004. Contribution à l'amélioration de la culture du teck (Tectona grandis L. f. Verbenaceae) en Côte d'Ivoire par l'évaluation d'un essai de descendance et par reproduction de clones. Mémoire de DEA de Botanique, UFR Biosciences, Université de Cocody Abidjan, p. 50.

Zézé A, Ouattara B, Brou CY, Van Tuinen D, Diallo-Attah H, Sangaré A. 2007. Distribution et abondance de spores de champignons endomycorhizogènes à arbuscules dans différents types de forêts de la Téné en côte d'ivoire. Agronomie Africaine, 19(2) : 103-111. 